\title{
APPLYING URBAN COMPACTNESS METRICS ON PAN-EUROPEAN DATASETS
}

\author{
D. Stathakis ${ }^{a, *}$, G. Tsilimigkas ${ }^{\text {b }}$ \\ ${ }^{a}$ University or Thessaly, Department of Planning and Regional Development, Pedion Areos, 38334, Volos, Hellenic \\ Republic,dstath@uth.gr \\ ${ }^{\mathrm{b}}$ University of Aegean, Department of Geography, University Hill, 81100, Mytilene, Hellenic Republic, \\ gtsil@geo.aegean.gr
}

KEY WORDS: Compact city, spatial metrics, Urban Atlas, Urban Audit.

\begin{abstract}
:
Urban compactness is measured for a number of medium sized European cities based on metrics available in the literature. The information used is a combination of Urban Atlas and Urban Audit data sets. The former is a source of spatial data whereas the latter of population data. These datasets that have been made recently available providing for the first time the opportunity to perform comparative analysis of urban compactness across European countries. The results provide an interesting insight of variation amongst cities in different countries. The analysis is limited however due to the quality and generalization of the datasets.
\end{abstract}

\section{INTRODUCTION}

The compact city is one of these concepts in planning that are hard to quantify (Burton 2000). The difficulty resides first of all in agreeing upon what exactly are the components of a compact city. But even if there is an exact answer to this question data availability in common standards would almost certainly force the measures to adapt from what should be measured but to what can be measured. Therefore, the quantification of a compact city is not an easy task. It is important however to be able to calculate urban compactness. The ability to measure it, as well as its change through time, is essential in monitoring cities. Even if intensification, i.e. increased compactness, is not planned but merely a spontaneous process planners should be able to have a clear view.

Compact city is an urban form that is alternative to urban sprawl (Besussi et al. 2010; Chin 2006). Compact city characteristics include high residential densities and increased mix of land uses as opposed to urban sprawl characterized by low densities and land use segregation (Neuman 2005). Some researches have proposed specific metrics to measure city compactness. Notably Burton (2002) proposed a metric of compactness that is composed of three poles (a) density (b) mix-of-use and (c) intensification. Each of these poles is measured as a combination of several indexes. The case study for this approach has been several medium sized cities in the UK. The objective of the present work is to try to apply a similar approach to European medium sized cities. Clearly the approach can only by partially applied due to data limitations based on the two standard datasets that cover the whole of the European Union, i.e. Urban Atlas and Urban Audit.

\section{DATA}

\subsection{Urban Atlas}

The spatial data for the cities come from Urban Atlas (European Union, 2011). It covers Large Urban Zones (LUZ) with more than 100,000 inhabitants. The data refer to the years 2005-7. It is suitable for 1:10,000 scale with a minimum mapping unit of 0.25 ha for artificial surfaces and 1 ha for all other classes. Its nominal thematic accuracy for the class referring to artificial surfaces is $85 \%$ or better. It is a product of interpretation of very high resolution earth observation data combined with locally available topographic and land use maps. Its original spatial structure is vector and it comes in Lambert Azimuthal Equal
Area projection which maps conveniently the European continent. A useful property of this projection is that it does not distort areas. Consequently it is suitable for calculating densities in a pan-European dataset. An important aspect of Urban Atlas is its classification system (Table 1) which aligns with CORINE's system after the addition of a finer level (forth level). Despite this hierarchical alignment with CORINE it is not easy to combine it with Urban Atlas in time series analysis due to the very different reference scales (Prastacos et al. 2012).

\begin{tabular}{ll} 
code & \multicolumn{1}{c}{ Land use } \\
\hline 11100 & Continuous Urban Fabric \\
\hline 11210 & Discontinuous Dense Urban Fabric \\
\hline 11220 & Discontinuous Medium Density Urban Fabric \\
\hline 11230 & Discontinuous Low Density Urban Fabric \\
\hline 11240 & Discontinuous Very Low Density Urban Fabric \\
\hline 11300 & Isolated structures \\
\hline 12100 & Industrial,commercial, public, military \& private units \\
\hline 12210 & Fast transit roads and associated land \\
\hline 12220 & Other roads and associated land \\
\hline 12230 & Railways and associated land \\
\hline 12300 & Port areas \\
\hline 12400 & Airports \\
\hline 13100 & Mineral extraction and dump sites \\
\hline 13300 & Construction sites \\
\hline 13400 & Land without current use \\
\hline 14100 & Green urban areas \\
\hline 14200 & Sports and leisure facilities \\
\hline 20000 & Agricultural areas, semi-natural areas and wetlands \\
\hline 30000 & Forests \\
\hline 50000 & Water \\
\hline
\end{tabular}

Table 1. Land use classes in Urban Atlas. 


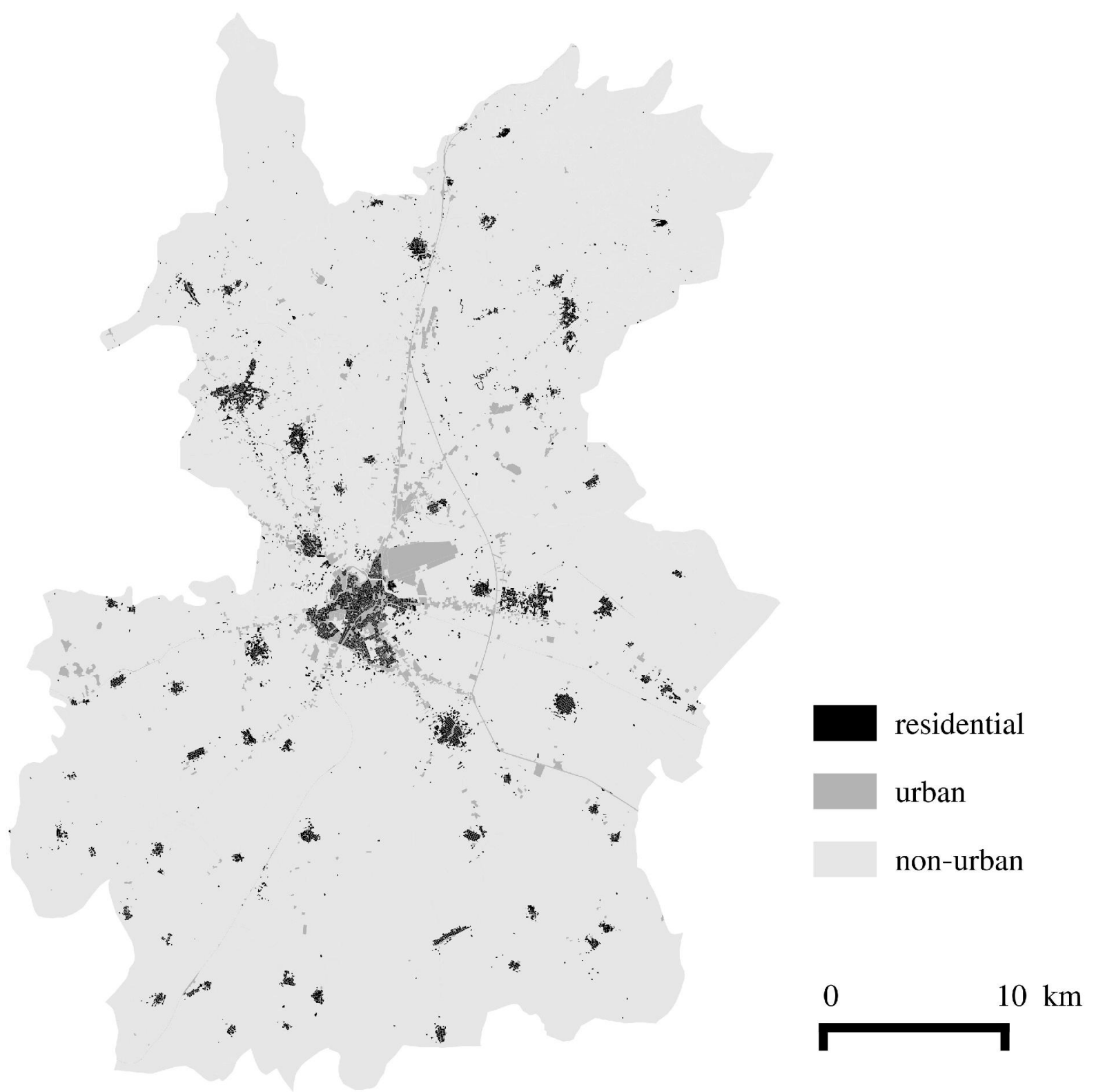

Figure 2. Urban Atlas data for the city of Larisa (gr005l_larisa).

In the following analysis Urban Atlas data was recoded to urban (codes $11100-14200)$, residential (codes $11100-11300$ ) and non-urban (codes 20000 - 50000). One example is shown in Figure 2.

\subsection{Urban Audit}

Urban Audit is a database containing statistical information for European cities. It has been coordinated by the statistical office of the European Union (Eurostat). One important aspect of Urban Audit is that it provides data also for Larger Urban Zones (LUZs). But although LUZs should coincide with the functional urban region its limits have been adapted to coincide with administrative boundaries. This is definitely convenient for gathering socioeconomic data it is nevertheless probably the source of some problems in the dataset discussed in the following section. An other problem is that the most recent date for obtaining the total resident population within a Larger
Urban Zone is the year 2004. For some cities the most recent year is even more distant. In specific, for all Bulgarian cities the most recent data is for 1991 whereas for Lincoln (UK) and Setubal (PT) it is 2001. Also other variables that would be useful in building indexes, such as the number of households and the number of dwellings, are not available for all the cities of the European Union.

\subsection{Data quality}

To a large extent the nominal accuracy of the datasets used in the study have been taken for granted in order to focus on the use of metrics. A systematic assessment of data quality is clearly out of the scope of the present work. However, there is evidence that some checking needs to be done in the future. To mention one example, the population of the LUZ of Volos (gr006l_volos) given in Urban Audit is significantly less compared to that given by official national statistics. An other problem is the delineation of urban zones in Urban Atlas. For example despite the fact that in terms of population the city of Ioannina (gr007l_ioannina) is seven times smaller compared to Thessaloniki (gr002l_thessaloniki) their zone acreage in Urban Atlas is surprisingly approximately the same. 


\section{METHOD}

\subsection{Selection of cities}

In any comparison between cities great differences in size can make results difficult to interpret. For that reason only medium sized cities are selected here for comparison. In specific the cities that qualify for comparison are those having a LUZ with total resident inhabitants between 80,000 and 220,000. The exact population limits are identical to those used by Burton (2002).

\subsection{Metrics}

The metrics used here are taken from Burton (2002). The author there assembles quite a large number of metrics. The data requirements for these metrics might be possible to be met at national scale, at least for some European countries, but is not possible to be met for a cross-European analysis. In fact by using the only European-wide available datasets, i.e. Urban Atlas and Urban Audit, only a few of these metrics can be used. In specific, and using the names proposed by Burton (2002), in terms of density only the following indexes can be used:

- $\quad$ densgr1 $=$ resident population in zone / total zone acreage (persons per hectare).

- $\quad$ densblt1 = residential population in zone / built-up acreage (persons per hectare). The correspondence of built-up area in Urban Atlas schema is given in section 2.1. Note that built-up includes residential.

- $\quad$ densres1 = resident population in zone / residential area acreage (persons per hectare).

With respect to the mix of use only the following index can be used:

- $\quad$ supfacs2 = residential $/$ non-residential urban land.

No metric can be used with respect to intensification, that is the change of city compactness throughout time. The reason is that currently both Urban Atlas and the Urban Audit do not offer a second point in time with pan-European coverage in order to estimate change.

The metrics have been standardized based on the following formula:

$$
\text { standardized value }=\frac{\text { value }- \text { mean }}{\text { standard deviation }}
$$

They were then combined to yield an overall density measure (dens) by averaging densblt1 and densres1. Index densgr1 is shown on Table 2 but has been omitted in calculating the overall density measure (dens). The reason is that, as explained in section 2.3 there is evidence that the delineation of LUZ is not consistent. The delineation of urban and residential areas is much more objective, especially when very high resolution satellite images are available, and thus more reliable. The overall mix-of-use index (mixuse) has only one component. Moreover, the intensification component is absent as already explained. An overall metric, termed compactness (compact), is calculated by averaging the overall density (dens) and mix-ofuse (mixuse) measures.

\section{RESULTS}

The results of the process are shown on Table 2 shorted on compactness. Population refers to total resident persons in the LUZ, zone area refers to total acreage of the LUZ, the correspondence of built-up and residential areas are given in section 2.1, code refers to Urban Atlas codes of cities and "compact" is the total metric of compactness. A more interesting view of the results is shown on figure 2 . In this scatterplot it becomes evident that the vast majority of the cities are clustered approximately around mean density and mix-ofuse values, i.e. where the two axes cross. There are however some interesting deviations.

Looking at the outliers in the rightmost part of this diagram, there is a cluster of values of over 2 or more standard deviations. This cluster is exclusively composed of Romanian cities with only one exception. This exception is the the most dens city namely Kavala (gr008). The other end of density is dominated by Swedish cities. Finally three cities are substantially more mixed compared to the average (pl020, hu003, pt004).

Overall the variation of compactness values at the European level is significant providing adequate contrast as opposed to data reported at national scale (Burton 2002). A further reason for this increased variation is perhaps that each pole of compactness (density and mix-of-use) has been calculated by very few components and thus the effect of averaging is inevitably minimized.

\section{CONCLUSIONS}

In conclusion the two datasets currently available can be used along with the metrics for the compact city that have already proposed to provide an insight on the current state of the cities. The two data sources are far from perfect. Nevertheless this is the only option currently available to anyone how would like to do a pan-European study of cities.

Only a few of the metrics originally proposed by Burton (2002) can be used with these data sources. The results are definitely limited in that respect. But this limitation is also valuable as a conclusion by itself. It shows that currently there are no reliable datasets to study European cities based on common standards.

The same finding can also be viewed differently. When studying European cities the metrics used should be more adapted to pan-European datasets rather than using methods that rely on country-specific datasets. Metrics could adapt to CORINE's and Urban Atlas' classes and scales. This is also an opportunity to shift the nature of metrics from statistical to spatial.

In addition, the realization of the lack of data to monitor European cities should also alert planners and policy makes since it is an indication that this field does not receive due attention despite of the fact that it is critical and that it affects the environment and the economy in multiple ways. This could mean that monitoring the cities should rely more on top down methods, such as remote sensing (Stathakis et al. 2012; Stathakis and Faraslis, to appear), rather than bottom-up data gathering.

An open research question is whether it is feasible to devise new metrics adapted to pan-European datasets that can better capture the dynamics of compactness and its evolution through time. That is currently a very interesting topic for further research. The other question is whether European institutions can become more active in producing reliable datasets for urban areas. 
International Archives of the Photogrammetry, Remote Sensing and Spatial Information Sciences,

Volume XL-4/W1, 29th Urban Data Management Symposium, 29 - 31 May, 2013, London, United Kingdom

\begin{tabular}{|c|c|c|c|c|c|c|c|c|c|c|c|c|}
\hline city & $\begin{array}{c}\text { population } \\
{[1]}\end{array}$ & $\begin{array}{c}\text { zone area (ha) } \\
\text { [2] }\end{array}$ & $\begin{array}{c}\text { Built-up (ha) } \\
\text { [3] }\end{array}$ & $\begin{array}{c}\text { Residential (ha) } \\
{[4]}\end{array}$ & $\begin{array}{c}\text { densgr } 1 \\
{[1] /[2]}\end{array}$ & $\begin{array}{l}\text { densblt1 } \\
{[5]=[1] /[3]}\end{array}$ & $\begin{array}{l}\text { densres } 1 \\
{[6]=[1] /[4]}\end{array}$ & $\begin{array}{c}\text { supfacs2 } \\
{[7]=[4] /([3]-[4])}\end{array}$ & code & $\begin{array}{c}\text { dens } \\
{[8]=([5]+[6]) / 2}\end{array}$ & $\begin{array}{c}\underset{[7]}{\operatorname{mixuse}} \\
\end{array}$ & $\begin{array}{c}\text { compact } \\
([8]+[7]) / 2\end{array}$ \\
\hline Nowy Sacz & 158,620 & 44,835 & 7,636 & 5,467 & 0.41 & $\quad 0.07$ & -0.61 & 4.39 & pl020 & -0.27 & 4.39 & 2.06 \\
\hline Targu Mures & 172,642 & 14,135 & 3,224 & 1,763 & 3.99 & 3.25 & 2.37 & 0.67 & roo10 & 2.81 & 0.67 & 1.74 \\
\hline Piatra Neamt & 124,194 & 14,671 & 2,561 & 1,452 & 2.44 & 2.76 & 1.84 & 0.96 & roo11 & 2.30 & 0.96 & 1.63 \\
\hline Funchal & 190,014 & 26,095 & 6,234 & 4,037 & 1.96 & 1.01 & 0.17 & 2.46 & pt004 & 0.59 & 2.46 & 1.52 \\
\hline Bacau & 203,559 & 22,078 & 4,235 & 2,246 & 2.76 & 2.72 & 2.06 & 0.45 & ro007 & 2.39 & 0.45 & 1.42 \\
\hline Kavala & 129,567 & 35,148 & 2,505 & 1,101 & 0.47 & 3.07 & 3.23 & -0.52 & gr008 & 3.15 & -0.52 & 1.31 \\
\hline Nyíregyháza & 218,153 & 143,765 & 17,861 & 12,109 & -0.42 & -0.76 & -1.09 & 3.21 & hu003 & -0.93 & 3.21 & 1.14 \\
\hline Iraklio & 202,426 & 60,447 & 6,179 & 3,399 & 0.33 & 1.23 & 0.71 & 0.72 & gro04 & 0.97 & 0.72 & 0.85 \\
\hline Oradea & 218,276 & 20,110 & 5,307 & 2,425 & 3.43 & 2.04 & 2.03 & -0.36 & ro006 & 2.04 & -0.36 & 0.84 \\
\hline Sibiu & 186,803 & 58,814 & 4,694 & 2,152 & 0.26 & 1.92 & 1.89 & -0.35 & rooog & 1.90 & -0.35 & 0.78 \\
\hline Alba Iulia & 97,745 & 25,882 & 3,053 & 1,643 & 0.51 & 1.16 & 0.71 & 0.55 & ro014 & 0.93 & 0.55 & 0.74 \\
\hline Pécs & 178,190 & 57,066 & 8,857 & 5,242 & 0.24 & 0.01 & -0.40 & 1.36 & hu004 & -0.20 & 1.36 & 0.58 \\
\hline Calarasi & 83,441 & 24,578 & 2,149 & 762 & 0.35 & 1.82 & 2.88 & -1.19 & ro012 & 2.35 & -1.19 & 0.58 \\
\hline Jelenia Góra & 127,382 & 58,565 & 6,267 & 3,587 & -0.15 & 0.03 & -0.33 & 1.05 & pl019 & -0.15 & 1.05 & 0.45 \\
\hline Arad & 189,099 & 51,978 & 6,623 & 3,350 & 0.45 & 0.82 & 0.58 & 0.15 & ro008 & 0.70 & 0.15 & 0.43 \\
\hline Namur & 139,024 & 39,741 & 9,424 & 5,640 & 0.39 & -0.51 & -0.80 & 1.47 & be 007 & -0.66 & 1.47 & 0.41 \\
\hline Zlin & 193,068 & 103,207 & 10,153 & 5,755 & -0.28 & -0.10 & -0.42 & 0.96 & cz011 & -0.26 & 0.96 & 0.35 \\
\hline Szeged & 197,417 & 75,292 & 10,799 & 6,154 & 0.03 & -0.17 & -0.48 & 1.01 & hu006 & -0.33 & 1.01 & 0.34 \\
\hline PRESOV & 163,743 & 93,455 & 8,012 & 4,355 & -0.33 & 0.04 & -0.24 & 0.63 & sk005 & -0.10 & 0.63 & 0.26 \\
\hline Nitra & 163,764 & 87,020 & 9,516 & 5,261 & -0.27 & -0.28 & -0.52 & 0.76 & sk004 & -0.40 & 0.76 & 0.18 \\
\hline Ajaccio & 83,026 & 101,502 & 6,502 & 3,740 & -0.71 & -0.71 & -0.91 & 1.09 & fro27 & -0.81 & 1.09 & 0.14 \\
\hline Suwalki & 82,539 & 61,833 & 4,211 & 2,227 & -0.50 & -0.04 & -0.26 & 0.43 & pl021 & -0.15 & 0.43 & 0.14 \\
\hline Setubal & 113,811 & 17,283 & 4,253 & 1,927 & 1.67 & 0.65 & 0.69 & -0.40 & pt006 & 0.67 & -0.40 & 0.14 \\
\hline Aveiro & 112,873 & 27,335 & 6,351 & 3,391 & 0.65 & -0.22 & -0.43 & 0.50 & pt008 & -0.32 & 0.50 & 0.09 \\
\hline Faro & 111,782 & 48,217 & 5,415 & 2,760 & -0.09 & 0.06 & -0.11 & 0.20 & pt009 & -0.03 & 0.20 & 0.09 \\
\hline Kecskemét & 170,452 & 148,304 & 16,229 & 9,348 & -0.58 & -0.93 & -1.08 & 1.10 & hu008 & -1.00 & 1.10 & 0.05 \\
\hline Catanzaro & 146,730 & 76,141 & 6,356 & 3,008 & -0.25 & 0.29 & 0.24 & -0.20 & it024 & 0.27 & -0.20 & 0.03 \\
\hline Ancona & 208,235 & 40,844 & 8,351 & 3,729 & 1.05 & 0.47 & 0.55 & -0.46 & it017 & 0.51 & -0.46 & 0.03 \\
\hline Žilina & 156,869 & 81,391 & 7,814 & 3,870 & -0.25 & 0.00 & -0.11 & 0.03 & sk006 & -0.06 & 0.03 & -0.01 \\
\hline Ruse & 201,410 & 89,261 & 7,374 & 2,664 & -0.12 & 0.70 & 1.41 & -1.14 & bg006 & 1.06 & -1.14 & -0.04 \\
\hline Foggia & 196,072 & 104,812 & 7,128 & 2,012 & -0.28 & 0.72 & 2.35 & -1.63 & it031 & 1.54 & -1.63 & -0.05 \\
\hline Valletta & 208,542 & 24,671 & 8,815 & 3,900 & 2.44 & 0.35 & 0.45 & -0.50 & $\mathrm{mt} 001$ & 0.40 & -0.50 & -0.05 \\
\hline Vidin & 85,974 & 51,750 & 4,462 & 2,181 & -0.36 & -0.08 & -0.16 & -0.04 & bg007 & -0.12 & -0.04 & -0.08 \\
\hline Ponto Delgada & 119,571 & 53,714 & 5,859 & 2,780 & -0.13 & 0.03 & -0.01 & -0.19 & pt007 & 0.01 & -0.19 & -0.09 \\
\hline Trento & 185,452 & 77,916 & 8,168 & 3,614 & -0.07 & 0.26 & 0.35 & -0.50 & it014 & 0.31 & -0.50 & -0.10 \\
\hline Gorzów Wielkopolski & 190,251 & 130,538 & 9,552 & 4,531 & -0.45 & -0.01 & -0.05 & -0.19 & pl017 & -0.03 & -0.19 & -0.11 \\
\hline Trencín & 112,515 & 67,404 & 6,002 & 2,877 & -0.36 & -0.13 & -0.17 & -0.14 & sk008 & -0.15 & -0.14 & -0.14 \\
\hline Banska Bystrica & 111,419 & 80,887 & 4,907 & 2,079 & -0.48 & 0.26 & 0.45 & -0.66 & sk003 & 0.35 & -0.66 & -0.15 \\
\hline Konin & 143,305 & 75,844 & 8,337 & 4,097 & -0.27 & -0.28 & -0.35 & -0.01 & pl022 & -0.32 & -0.01 & -0.16 \\
\hline Hradec Kralove & 159,293 & 87,600 & 10,146 & 5,030 & -0.30 & -0.42 & -0.50 & 0.04 & cz009 & -0.46 & 0.04 & -0.21 \\
\hline Székesfehérvár & 155,877 & 114,417 & 11,685 & 6,031 & -0.49 & -0.65 & -0.75 & 0.28 & hu009 & -0.70 & 0.28 & -0.21 \\
\hline Campobasso & 116,507 & 130,868 & 9,613 & 5,049 & -0.68 & -0.77 & -0.87 & 0.39 & it020 & -0.82 & 0.39 & -0.22 \\
\hline Apeldoorn & 212,948 & 62,505 & 11,832 & 5,551 & 0.36 & -0.20 & -0.21 & -0.24 & nl014 & -0.20 & -0.24 & -0.22 \\
\hline Santiago de Compostelé & 186,332 & 135,165 & 17,203 & 9,168 & -0.48 & -0.90 & -0.99 & 0.49 & es011 & -0.94 & 0.49 & -0.23 \\
\hline Perugia & 207,569 & 80,602 & 11,612 & 5,433 & 0.01 & -0.21 & -0.21 & -0.25 & it016 & -0.21 & -0.25 & -0.23 \\
\hline Brugge & 165,743 & 41,228 & 10,260 & 4,953 & 0.61 & -0.38 & -0.42 & -0.10 & be006 & -0.40 & -0.10 & -0.25 \\
\hline Pleven & 206,936 & 179,197 & 11,400 & 5,236 & -0.57 & -0.18 & -0.16 & -0.34 & bg005 & -0.17 & -0.34 & -0.25 \\
\hline Sassari & 200,554 & 122,648 & 12,326 & 5,903 & -0.37 & -0.37 & -0.40 & -0.14 & it026 & -0.38 & -0.14 & -0.26 \\
\hline Leeuwarden & 158,883 & 45,205 & 7,870 & 3,313 & 0.40 & 0.01 & 0.21 & -0.69 & nl015 & 0.11 & -0.69 & -0.29 \\
\hline Zielona Góra & 207,451 & 162,701 & 11,222 & 4,862 & -0.52 & -0.15 & -0.02 & -0.58 & $\mathrm{pl018}$ & -0.09 & -0.58 & -0.33 \\
\hline Koszalin & 171,469 & 175,106 & 9,248 & 3,983 & -0.65 & -0.15 & 0.00 & -0.60 & pl028 & -0.08 & -0.60 & -0.34 \\
\hline Lincoln & 164,418 & 72,378 & 10,076 & 4,528 & -0.11 & -0.36 & -0.30 & -0.43 & uk019 & -0.33 & -0.43 & -0.38 \\
\hline Tartu & 148,872 & 300,056 & 15,269 & 7,858 & -0.85 & -1.00 & -1.05 & 0.26 & ee002 & -1.02 & 0.26 & -0.38 \\
\hline TRNAVA & 126,822 & 74,092 & 7,678 & 3,412 & -0.34 & -0.34 & -0.26 & -0.48 & sk007 & -0.30 & -0.48 & -0.39 \\
\hline Pardubice & 159,981 & 89,091 & 11,266 & 5,187 & -0.31 & -0.57 & -0.53 & -0.33 & cz010 & -0.55 & -0.33 & -0.44 \\
\hline Jihlava & 108,292 & 117,869 & 7,668 & 3,518 & -0.67 & -0.58 & -0.54 & -0.34 & cz014 & -0.56 & -0.34 & -0.45 \\
\hline l'Aquila & 100,592 & 158,778 & 8,637 & 4,165 & -0.79 & -0.82 & -0.82 & -0.11 & it018 & -0.82 & -0.11 & -0.46 \\
\hline Ceske Budejovice & 179,369 & 162,455 & 13,847 & 6,440 & -0.59 & -0.69 & -0.66 & -0.28 & cz008 & -0.68 & -0.28 & -0.48 \\
\hline Poitiers & 216,847 & 176,075 & 21,018 & 10,144 & -0.54 & -0.95 & -0.94 & -0.10 & fro21 & -0.94 & -0.10 & -0.52 \\
\hline Panevezys & 160,656 & 222,822 & 12,981 & 5,972 & -0.75 & -0.75 & -0.70 & -0.33 & It003 & -0.72 & -0.33 & -0.53 \\
\hline Weimar & 153,353 & 88,949 & 9,241 & 3,669 & -0.34 & -0.34 & -0.06 & -0.88 & de030 & -0.20 & -0.88 & -0.54 \\
\hline Örebro & 178,748 & 368,777 & 22,975 & 11,409 & -0.85 & -1.19 & -1.19 & 0.05 & se008 & -1.19 & 0.05 & -0.57 \\
\hline Logroño & 171,599 & 143,702 & 9,401 & 2,232 & -0.56 & -0.17 & 1.46 & -1.86 & es018 & 0.64 & -1.86 & -0.61 \\
\hline Oulu & 196,096 & 376,836 & 21,387 & 10,179 & -0.84 & -1.06 & -1.03 & -0.17 & fio04 & -1.05 & -0.17 & -0.61 \\
\hline Potenza & 145,337 & 149,847 & 11,470 & 4,948 & -0.65 & -0.72 & -0.60 & -0.60 & it023 & -0.66 & -0.60 & -0.63 \\
\hline Cremona & 132,159 & 66,133 & 8,132 & 2,892 & -0.22 & -0.37 & 0.11 & -1.18 & it013 & -0.13 & -1.18 & -0.66 \\
\hline Ioannina & 139,522 & 132,632 & 9,364 & 3,575 & -0.62 & -0.50 & -0.18 & -1.00 & groot & -0.34 & -1.00 & -0.67 \\
\hline Larissa & 187,831 & 155,570 & 11,651 & 4,023 & -0.55 & -0.38 & 0.15 & -1.25 & gr005 & -0.11 & -1.25 & -0.68 \\
\hline Liepaja & 131,788 & 365,731 & 11,136 & 4,529 & -0.90 & -0.80 & -0.61 & -0.80 & Iv002 & -0.70 & -0.80 & -0.75 \\
\hline Umeấ & 139,588 & 981,210 & 25,950 & 12,494 & -0.99 & -1.42 & -1.39 & -0.11 & se005 & -1.40 & -0.11 & -0.76 \\
\hline Jönköping & 148,693 & 347,332 & 18,894 & 8,138 & -0.87 & -1.18 & -1.08 & -0.60 & se004 & -1.13 & -0.60 & -0.87 \\
\hline Linköping & 183,221 & 423,193 & 23,690 & 9,989 & -0.87 & -1.20 & -1.07 & -0.68 & se007 & -1.14 & -0.68 & -0.91 \\
\hline Karlovy Vary & 121,430 & 162,137 & 11,246 & 4,109 & -0.74 & -0.90 & -0.59 & -1.11 & cz013 & -0.74 & -1.11 & -0.93 \\
\hline Toledo & 167,036 & 361,635 & 15,387 & 4,411 & -0.86 & -0.89 & -0.23 & -1.61 & es016 & -0.56 & -1.61 & -1.08 \\
\hline
\end{tabular}

Table 2. Compactness and other data per LUZ. The most recent population data for Setubal and Lincoln is 2001 whereas for Pleven, Vidin and Ruse is 1991 
International Archives of the Photogrammetry, Remote Sensing and Spatial Information Sciences,

Volume XL-4/W1, 29th Urban Data Management Symposium, 29 - 31 May, 2013, London, United Kingdom

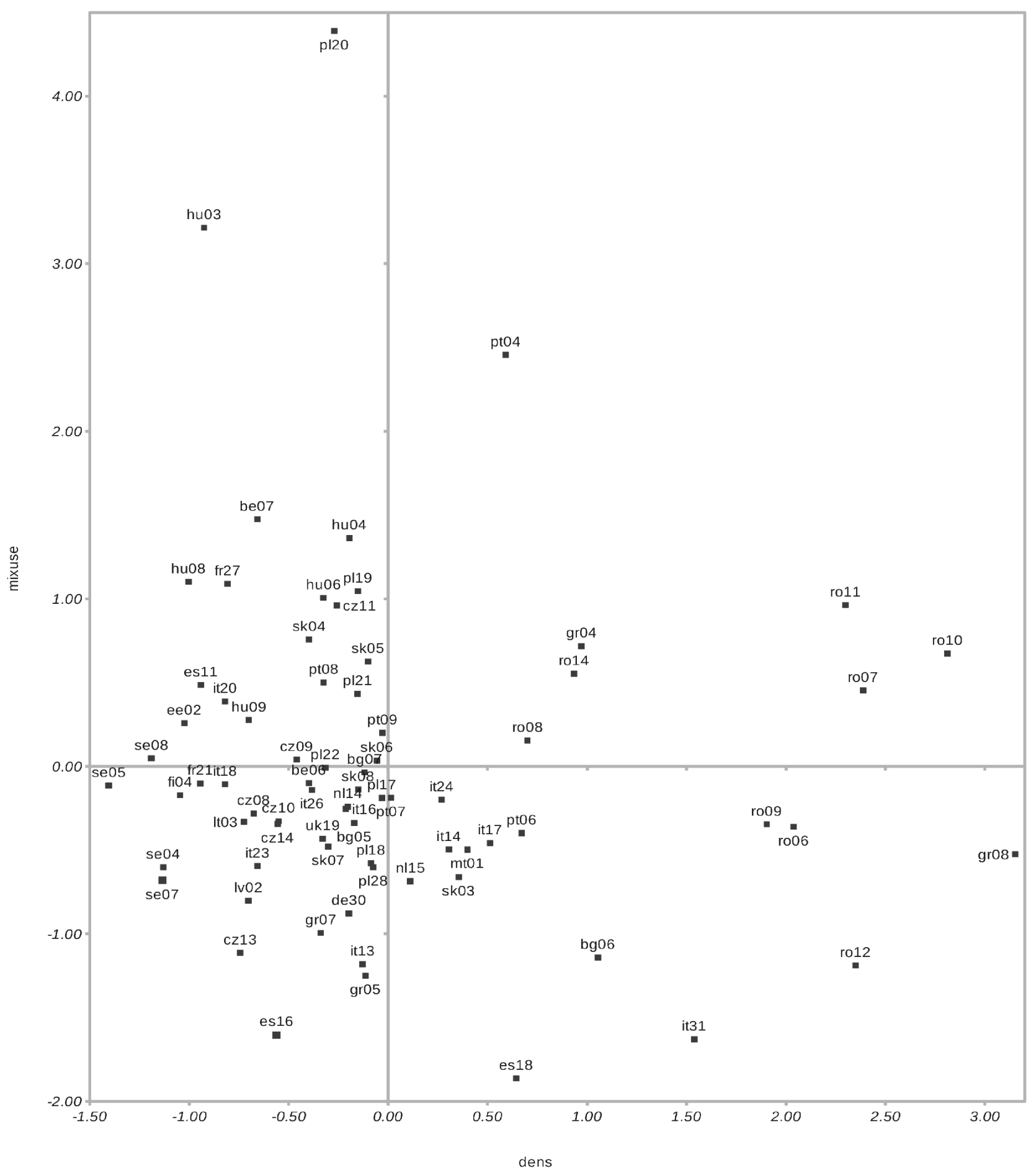

Figure 2. Compactness of cities based on density and mix-ofuse. 


\section{ACKNOWLEDGEMENT}

The Urban Atlas dataset is a property of the European Commission (Directorate-General Enterprise and Industry and Directorate-General for Regional Policy).

\section{REFERENCES}

Besussi E, Chin N, Batty M, and P. Longley , 2010, The Structure and Form of Urban Settlements. In: Rashed $\mathrm{T}$ and Jürgens C (eds) Remote Sensing of Urban and Suburban Areas. Netherlands: Springer, pp. 13-31.

Burton, E. 2000. The Compact City: Just or Just Compact? A Preliminary Analysis. Urban Studies, (37)11, pp. 1969-2001.

Burton, E. 2002. Measuring urban compactness in UK towns and cities. Environment and Planning B: Planning and Design (29), pp. 219-250.

Chin NNG., 2006, Spatial analysis and the measurement of urban sprawl, Centre for Advanced Spatial Analysis and Geography Department. London: University College London, pp. 346.

European Union, 2011. Mapping Guide for a European Urban Atlas. Directorate-General for Regional Policy: 1 - 31 . available at

http://www.eea.europa.eu/data-and-maps/data/urban-atlas/

Prastacos, P., Chrysoulakis N., and Kochilakis G., 2012, Spatial metrics for Greek cities using land cover information from the Urban Atlas, Proceedings of the AGILE'2012 International Conference on Geographic Information Science, Avignon, April, 24-27. Multidisciplinary Research on Geographical Information in Europe and Beyond, Editors: Jérôme Gensel, Didier Josselin and Danny Vandenbroucke, pp. 261-266

Neuman M., 2005, The Compact City Fallacy, Journal of Planning Education and Research. 25, pp. 11-26.

Stathakis D., K. Perakis and I. Savin, 2012, Efficient segmentation of urban areas by the VIBI, International Journal of Remote Sensing, vol. 33, no. 20, October, pp. 6361 - 6377.

Stathakis D., and I. Faraslis, to appear, Monitoring urban sprawl using simulated Proba-V data, International Journal of Remote Sensing.

Revised March 2013 